\title{
Distribution characteristics and influencing factors of the frequency-domain response of a vehicle-track vertical coupled system
}

\author{
Jinhui Xu ${ }^{1} \cdot$ Biao Wang ${ }^{1} \cdot$ Li Wang ${ }^{1} \cdot$ Ping Wang ${ }^{1}$
}

Received: 29 May 2015/Revised: 24 May 2016/Accepted: 26 May 2016/Published online: 18 June 2016

(C) The Author(s) 2016. This article is published with open access at Springerlink.com

\begin{abstract}
Employing theory on vehicle-track coupled dynamics, the equation of motion of a vehicle-track vertical coupled system was established by combining frequency analysis and symplectic mathematics. The frequency response of the vehicle-track vertical coupled system was calculated under the excitation of the German low-interference spectrum, and the effects of the vehicle speed, vehicle suspension parameters, and track support parameters on the frequency response of the coupled system were studied. Results show that, under the excitation of the German lowinterference spectrum, the vertical vibration of the car body is mainly concentrated in the low-frequency band, while that of the bogie has a wide frequency distribution, being strong from several Hertz to dozens of Hertz. The vertical vibrations of the wheel-rail force, wheelset, and track structure mainly occur at a frequency of dozens of Hertz. In general, the vertical vibration of the vehicle-track coupled system increases with vehicle speed, and the vertical vibrations of the car body and bogie obviously shift to higher frequency. Increasing the vehicle suspension stiffness increases the lowfrequency vibrations of the vehicle system and track structure. With an increase in vehicle suspension damping, the low-frequency vibrations of the car body and bogie and the
\end{abstract}

Biao Wang

601782752@qq.com

Jinhui Xu

491202515@qq.com

Li Wang

295289362@qq.com

Ping Wang

wping@home.swjtu.edu.cn

1 MOE Key Laboratory of High-Speed Railway Engineering, Southwest Jiaotong University, Chengdu 6100131, China vibrations of the wheel-rail vertical force and track structure decrease at $50-80 \mathrm{~Hz}$, while the mid-frequency and highfrequency vibrations of the car body and bogie increase. Similarly, an increase in track stiffness amplifies the vertical vibrations of the wheel-rail force and track structure, while an increase in track damping effectively reduces the vertical vibrations of the wheel-rail vertical force and track structure.

Keywords Vehicle-track coupled dynamics · Frequency response - Distribution characteristic - Influencing factors

\section{Introduction}

Track irregularity is the main excitation source causing the vibration of vehicle-track structures. Previous studies [1,2] have shown that track irregularity is a random process that cannot be described with deterministic functions. Vibrations of vehicle-track coupled systems excited by track irregularities should therefore be addressed using random vibration theory.

At present, two kinds of analyses are available to analyze the random vibration of a vehicle-track coupled system: time-domain analysis and frequency-domain analysis. Time-domain analysis uses a step-by-step integration method to solve the dynamic response under track irregularities measured or simulated using the track irregularity spectrum. By conducting time-domain analysis, Chen [3] and Zhai [4] analyzed the effect of track irregularity on the vehicle-track coupled dynamic response based on an analysis model for the vehicle-track coupled system. Lei and Noda [5] analyzed the dynamic response of a vehicletrack coupled system using the finite element method and studied the effects of the vehicle speed and track parameters on the vibration of the coupled system. Time-domain 
analysis can deal with nonlinear factors, but it needs a sufficient length of track irregularity samples and small integration steps, resulting in low calculation efficiency.

Frequency-domain analysis is another common type of random vibration analysis [6]. Many studies [7-9] on the random vibration of a vehicle-track system have employed frequency-domain analysis. To improve the efficiency of computation, Lin and Zhang [10] put forward a pseudoexcitation method. The pseudo-excitation method was then adopted by $\mathrm{Lu}$ et al. [11] and Zhang et al. [12, 13] to analyze the vertical random vibration of a vehicle-track coupled system. This method regards the track structure as a periodic structure and solves the spreading of harmonic virtual track irregularity excitation in the track substructure with a symplectic mathematics method. This method needs only four stress track substructures in the calculation, which greatly reduces the number of degrees of freedom (DOFs) and greatly improves the calculation efficiency. Employing the pseudo-excitation method, Zhang et al. [14] studied the random vibration of the vehicle-slab track coupled system and analyzed the effects of the vehicle speed and slab track parameters on the random vibration of the system.

The present work establishes a model for the vehicletrack vertical coupled system based on the theory of vehicle-track coupled dynamics. With this model, the frequency response of the vehicle-track vertical coupled system is calculated, and the effects of the vehicle speed, vehicle suspension parameters, and track support parameters on the frequency response of the coupled system are analyzed.

\section{Frequency analysis method for the vehicle-track vertical coupled system}

In many traditional dynamic analysis models, the vehicle system is regarded as a multi-rigid-body system $[3,4,11]$. In recent years, many scholars [15-18] have studied the effect of an elastic car body on the vibration of a vehicletrack coupled system. Studies $[17,18]$ found an important effect of the elastic car body on the riding comfort, but the distribution rules in the frequency domain of the vibration response calculated using the elastic car body model are similar to those calculated using the rigid car body model.

The dynamic model of a vehicle-track vertical coupled system is shown in Fig. 1, which includes a vehicle subsystem and a track subsystem connected by the interaction between wheels and track. The vehicle is regarded as a multi-rigid-body system composed of a car body, bogie frame, wheelset, suspension springs, and damping system, with 10 DOFs. A three-tier support model is adopted for

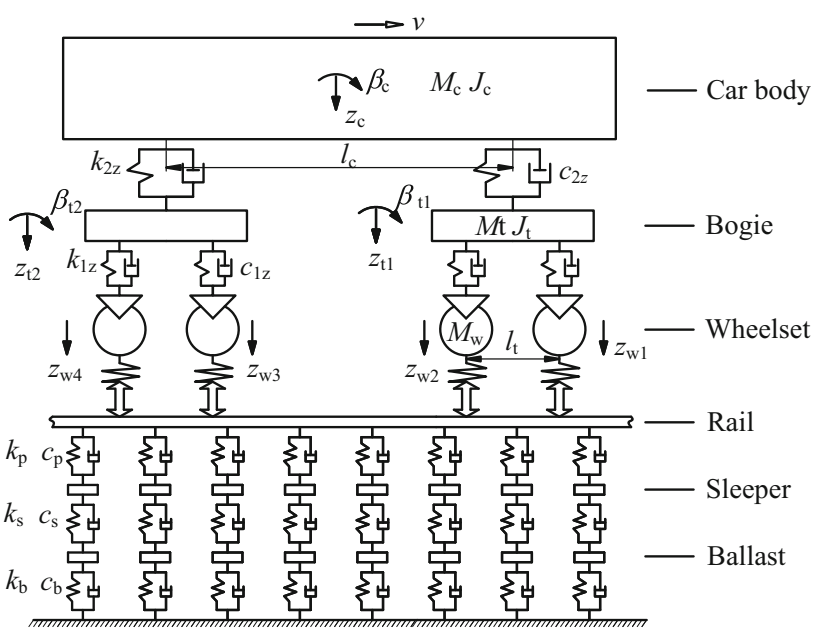

Fig. 1 Vehicle-track vertical coupled dynamic model

the track structure. A rail is regarded as a Euler beam, and sleepers and ballast are regarded as rigid mass blocks.

In Fig. $1, M_{\mathrm{c}}, M_{\mathrm{t}}$, and $M_{\mathrm{w}}$ are the masses of the car body, bogie, and wheelset, respectively; $J_{\mathrm{c}}$ and $J_{\mathrm{t}}$ are the rotational inertia of the car body and bogie, respectively; $k_{1 z}$ and $k_{2 z}$ are values of the vertical stiffness of the primary and secondary suspensions, respectively; $c_{1 z}$ and $c_{2 z}$ are the damping coefficients of the primary and secondary suspensions, respectively; $\beta_{\mathrm{c}}$ and $\beta_{\mathrm{t} i}$ are the roll angles of the car body and $i$ th bogie $(i=1,2)$, respectively; $z_{\mathrm{c}}, z_{\mathrm{t}}$, and $z_{\mathrm{w} j}$ are the vertical displacements of the car body, the $i$ th bogie $(i=1,2)$, and the $j$ th wheel $(j=1,2,3,4)$, respectively; $l_{\mathrm{t}}$ is the length of the fixed wheelbase, while $l_{\mathrm{c}}$ is the length of the distance between the two bogies; $k_{\mathrm{p}}, k_{\mathrm{s}}$, and $k_{\mathrm{b}}$ are the values of the vertical stiffness of the fastener system, ballast, and subgrade, respectively; and $c_{\mathrm{p}}, c_{\mathrm{s}}$, and $c_{\mathrm{b}}$ are the respective damping coefficients.

The vehicle subsystem and track subsystem are connected by wheel-track contact. The wheel-rail vertical force can be determined using Hertz contact theory. All parameters in frequency-domain analysis should be linear, and an equivalent linearization of the wheel-track nonlinear contact springs is thus required [3]. The linearized stiffness $k_{\mathrm{h}}$ of the Hertz spring is

$k_{\mathrm{h}}=\frac{2 p_{0}^{1 / 3}}{2 G}$,

where $G$ is a constant that depends on the wheel radius and material properties, and $P_{0}$ is the static wheel-rail force.

\subsection{Equation of motion of the vehicle system}

The equation of motion of the vehicle system is

$\boldsymbol{M}_{\mathrm{v}} \ddot{z}+\boldsymbol{C}_{\mathrm{v}} \dot{z}+\boldsymbol{K}_{\mathrm{v}} z=\boldsymbol{p}_{\mathrm{v}}$, 
where $z$ is the displacement vector of the vehicle system; $\boldsymbol{M}_{\mathrm{v}}, \boldsymbol{C}_{\mathrm{v}}$ and $\boldsymbol{K}_{\mathrm{v}}$ are the vehicle mass, damping, and rigidity matrixes, respectively; and $\boldsymbol{p}_{\mathrm{v}}$ is the wheel-rail force vector acting on the vehicle system.

A Fourier transform of the above equation provides the frequency-domain equation of motion of the vehicle system:

$\left(-\omega^{2} \boldsymbol{M}_{\mathrm{v}}+\mathrm{i} \omega \boldsymbol{C}_{\mathrm{v}}+\boldsymbol{K}_{\mathrm{v}}\right) \boldsymbol{u}_{\mathrm{v}}=\boldsymbol{f}_{\mathrm{v}}$,

where $\boldsymbol{u}_{\mathrm{v}}$ is the displacement vector of the vehicle system in the frequency domain; $f_{\mathrm{v}}$ is the wheel-rail force vector in the frequency domain; $\omega$ is the angular frequency; and i is the imaginary unit.

\subsection{Equation of motion of the track system}

The track structure is regarded as a periodic structure, and the part between two sleepers is taken as its substructure [12], as shown in Fig. 2. The track substructure has six DOFs, including two DOFs on each of the ends of the rail beam elements $\left(z_{\mathrm{r} 1}, \theta_{\mathrm{r} 1} ; z_{\mathrm{r} 2}, \theta_{\mathrm{r} 2}\right)$ and two vertical DOFs on the sleeper and ballast blocks $\left(z_{\mathrm{s}}\right.$ and $\left.z_{\mathrm{b}}\right)$, where $z$ is the vertical displacement of the track substructure and $\theta$ is the roll angle of rail beam elements, and subscripts $\mathrm{r} 1$ and $\mathrm{r} 2$ denote that the variables are associated with the two rail ends, the subscript $\mathrm{s}$ denotes a variable associated with the sleeper, and the subscript $b$ denotes a variable associated with the ballast.

The equation of motion of the loaded track substructure is

$\boldsymbol{M}_{\mathrm{t}} \ddot{\boldsymbol{u}}_{\mathrm{t}}+\boldsymbol{C}_{\mathrm{t}} \dot{\boldsymbol{u}}_{\mathrm{t}}+\boldsymbol{K}_{\mathrm{t}} \boldsymbol{u}_{\mathrm{t}}=\boldsymbol{p}_{\mathrm{t}}$,

where $\boldsymbol{u}_{\mathrm{t}}$ is the displacement vector of the track substructure; $\boldsymbol{M}_{\mathrm{t}}, \boldsymbol{C}_{\mathrm{t}}$ and $\boldsymbol{K}_{\mathrm{t}}$ are, respectively, the mass, damping, and stiffness matrixes of the track substructure; and $\boldsymbol{p}_{\mathrm{t}}$ is the wheel-rail force vector acting on the track substructure.

Applying a Fourier transform to the above equation, we obtain the frequency-domain equation of the loaded track substructure in block matrix form:

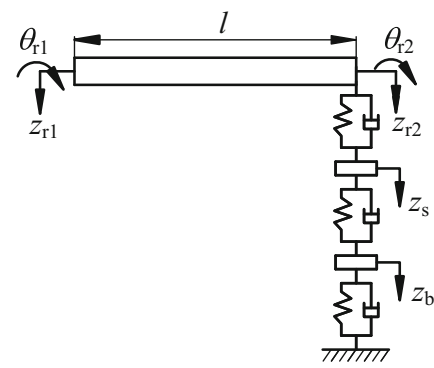

Fig. 2 Model of the track substructure

$$
\begin{aligned}
\left(-\omega^{2} \boldsymbol{M}_{\mathrm{t}}+\mathrm{i} \omega \boldsymbol{C}_{\mathrm{t}}+\boldsymbol{K}_{\mathrm{t}}\right) \boldsymbol{u}_{\mathrm{t}} & =\left[\begin{array}{lll}
\boldsymbol{k}_{11} & \boldsymbol{k}_{12} & \boldsymbol{k}_{13} \\
\boldsymbol{k}_{21} & \boldsymbol{k}_{22} & \boldsymbol{k}_{23} \\
\boldsymbol{k}_{31} & \boldsymbol{k}_{32} & \boldsymbol{k}_{33}
\end{array}\right]\left[\begin{array}{l}
\boldsymbol{u}_{\mathrm{t} 1} \\
\boldsymbol{u}_{\mathrm{t} 2} \\
\boldsymbol{u}_{\mathrm{t} 3}
\end{array}\right] \\
& =-f_{\mathrm{w}} \boldsymbol{N}_{\mathrm{h}}^{\mathrm{T}}+\left[\begin{array}{c}
\boldsymbol{f}_{\mathrm{tr} 1} \\
\boldsymbol{f}_{\mathrm{tr} 2} \\
0
\end{array}\right]
\end{aligned}
$$

where $\boldsymbol{k}_{i j}(i, j=1,2,3)$ is the block matrix of dynamic stiffness matrix; $\boldsymbol{u}_{\mathrm{t} 1}=\left(z_{\mathrm{r} 1}, \theta_{\mathrm{r} 1}\right)^{\mathrm{T}}$ is the displacement vector of the left section of the track substructure; $\boldsymbol{u}_{\mathrm{t} 2}=\left(z_{\mathrm{r} 2}, \theta_{\mathrm{r} 2}\right)^{\mathrm{T}}$ is the displacement vector of the right section of the track substructure; $\boldsymbol{u}_{\mathrm{t} 3}=\left(z_{\mathrm{s}}, \mathrm{z}_{\mathrm{b}}\right)^{\mathrm{T}}$ is the displacement vector of the internal DOF of the track substructure; $f_{\mathrm{w}}$ is the wheel-rail force; $N_{\mathrm{h}}=\left(N_{1}, N_{2}, N_{3}\right.$, $\left.N_{4}, 0,0\right)$ is the shape function vector; and $\boldsymbol{f}_{\text {tr } 1}$ and $f_{\text {tr2 } 2}$ are forces acting on the left and right neighboring substructures, respectively.

For an unloaded substructure $f_{\mathrm{w}}=0$, by removing $\boldsymbol{u}_{\mathrm{t} 3}$ in Eq. (5), the equation of motion of an unloaded track substructure can be written in vector form as

$\left[\begin{array}{c}\boldsymbol{u}_{\mathrm{t} 2} \\ \boldsymbol{f}_{\mathrm{tr} 2}\end{array}\right]=\boldsymbol{S}\left[\begin{array}{c}\boldsymbol{u}_{\mathrm{t} 1} \\ \boldsymbol{f}_{\mathrm{tr} 1}\end{array}\right]$

where $S$ is a transfer matrix that meets the symplectic orthogonal relationship. The stiffness matrixes $\boldsymbol{P}_{\alpha}$ and $\boldsymbol{P}_{\beta}$ of neighboring substructures can be obtained from the symplectic characteristics of the transfer matrix of unstressed substructures [11]. Inserting the stiffness matrixes $\boldsymbol{P}_{\alpha}$ and $\boldsymbol{P}_{\beta}$ into Eq. (5), we have

$\boldsymbol{k}_{\mathrm{tr}} \boldsymbol{u}_{\mathrm{tr}}=-\boldsymbol{N}^{\mathrm{T}} f_{\mathrm{w}}$,

where $\boldsymbol{k}_{\text {tr }}$ is the dynamic stiffness matrix of the loaded track substructure and $\boldsymbol{N}=\left(N_{1}, N_{2}, N_{3}, N_{4}\right)$ is the shape function vector of the Euler beam.

The overall equation of motion of the four loaded track substructures can be obtained from Eq. (7) as

$$
\begin{aligned}
& {\left[\begin{array}{llll}
\boldsymbol{k}_{\mathrm{tr} 1} & & & \\
& \boldsymbol{k}_{\mathrm{tr} 2} & & \\
& & \boldsymbol{k}_{\mathrm{tr} 3} & \\
& & & \boldsymbol{k}_{\mathrm{tr} 4}
\end{array}\right]\left[\begin{array}{l}
\boldsymbol{u}_{\mathrm{tr} 1} \\
\boldsymbol{u}_{\mathrm{tr} 2} \\
\boldsymbol{u}_{\mathrm{tr} 3} \\
\boldsymbol{u}_{\mathrm{tr} 4}
\end{array}\right]} \\
& =-\left[\begin{array}{llll}
\boldsymbol{N}\left(\xi_{1}\right) & & & \\
& \boldsymbol{N}\left(\xi_{2}\right) & & \\
& & \boldsymbol{N}\left(\xi_{3}\right) & \\
& & & \boldsymbol{N}\left(\xi_{4}\right)
\end{array}\right]^{\mathrm{T}}\left[\begin{array}{l}
f_{\mathrm{w} 1} \\
f_{\mathrm{w} 2} \\
f_{\mathrm{w} 3} \\
f_{\mathrm{w} 4}
\end{array}\right],
\end{aligned}
$$

where $\xi_{i}(i=1,2,3,4)$ is the distance between the $i$ th railwheel contact point and the left section of this loaded substructure.

\subsection{Wheel-rail coupled}

The vehicle and track are connected by linear Hertz contact springs, and the wheel-rail force can be expressed as 
Table 1 Parameters of the vehicle system

\begin{tabular}{lll}
\hline Parameter & Symbol & Value \\
\hline Car body mass & $M_{\mathrm{c}}$ & $39,080 \mathrm{~kg}$ \\
Bogie mass & $M_{\mathrm{t}}$ & $3,060 \mathrm{~kg}$ \\
Wheelset mass & $M_{\mathrm{w}}$ & $1,200 \mathrm{~kg}$ \\
Car body pitch inertia & $J_{\mathrm{c}}$ & $2.1 \times 10^{6} \mathrm{~kg} \cdot \mathrm{m}^{2}$ \\
Bogie pitch inertia & $J_{\mathrm{t}}$ & $3.2 \times 10^{3} \mathrm{~kg} \cdot \mathrm{m}^{2}$ \\
Primary suspension stiffness & $k_{1 \mathrm{z}}$ & $2.0 \times 10^{6} \mathrm{~N} / \mathrm{m}$ \\
Primary suspension damping & $c_{1 z}$ & $5.0 \times 10^{4} \mathrm{~N} \cdot \mathrm{s} / \mathrm{m}$ \\
Secondary suspension stiffness & $k_{2 z}$ & $4.06 \times 10^{5} \mathrm{~N} / \mathrm{m}$ \\
Secondary suspension damping & $c_{2 z}$ & $1.0 \times 10^{5} \mathrm{~N} \cdot \mathrm{s} / \mathrm{m}$ \\
Length between bogie pivot centers & $l_{\mathrm{c}}$ & $17.5 \mathrm{~m}$ \\
Fixed wheelbase & $l_{\mathrm{t}}$ & $2.5 \mathrm{~m}$ \\
Wheel radius & $r_{\mathrm{w}}$ & $0.4575 \mathrm{~m}$ \\
\hline
\end{tabular}

$f_{\mathrm{w} i}=k_{\mathrm{h}}\left(u_{\mathrm{r} i}+z_{\mathrm{ri}}-u_{\mathrm{w} i}\right)$,

where $u_{\mathrm{r} i}$ is the rail displacement at the $i$ th point of the wheel-rail force, $z_{\text {ri }}$ is the track irregularity at the $i$ th point of the wheel-rail force, $u_{\mathrm{w} i}$ is the displacement of the $i$ th wheelset, and $i=1,2,3,4$.

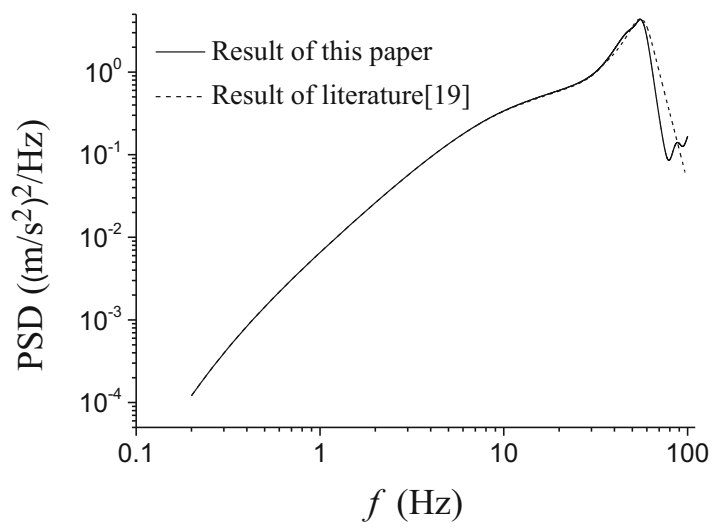

(a) Vertical acceleration of the wheelset

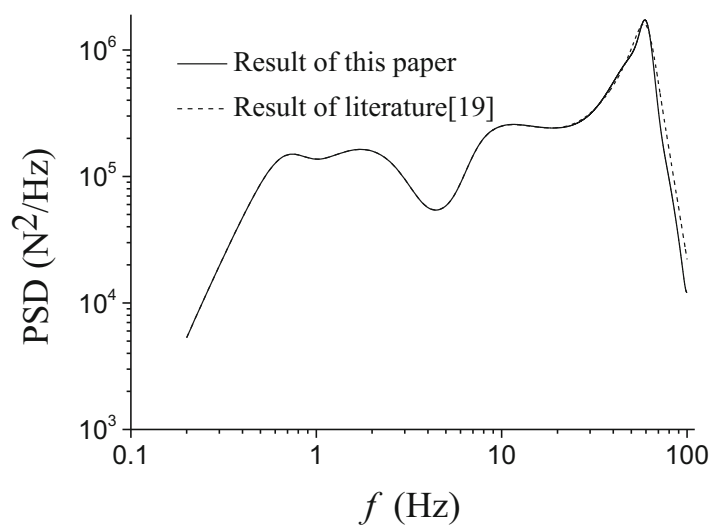

(c) Wheel-rail vertical force
From Eqs. (3), (8), and (9), the equation of motion of the coupled system can be obtained as

$\boldsymbol{K} \boldsymbol{U}=\boldsymbol{K}_{\mathrm{r}} \boldsymbol{Z}_{\mathrm{r}}$

where $\boldsymbol{K}$ is the stiffness matrix of the coupled system; $\boldsymbol{U}$ is the displacement vector of the coupled system; $\boldsymbol{K}_{\mathrm{r}}$ is the excitation coefficient matrix; and $\boldsymbol{Z}_{\mathrm{r}}$ is the system excitation input vector.

It is known from random vibration theory of a linear system that, if the system excitation is $\mathrm{e}^{\mathrm{i} \omega t}$, the system response is $\boldsymbol{H}(\omega) \mathrm{e}^{\mathrm{i} \omega t}$, where $\boldsymbol{H}(\omega)$ is the frequency response function of the system. Equation (10) can be rewritten as

$\boldsymbol{K} \boldsymbol{H}=\boldsymbol{K}_{\mathrm{r}}$,

$\boldsymbol{H}=\boldsymbol{K}^{-1} \boldsymbol{K}_{\mathrm{r}}$.

The frequency response function between track irregularities and the vibration response of the vehicletrack vertical coupled system can be obtained from Eq. (12). According to the random vibration theory, the excitation and response have the relationship as follows:

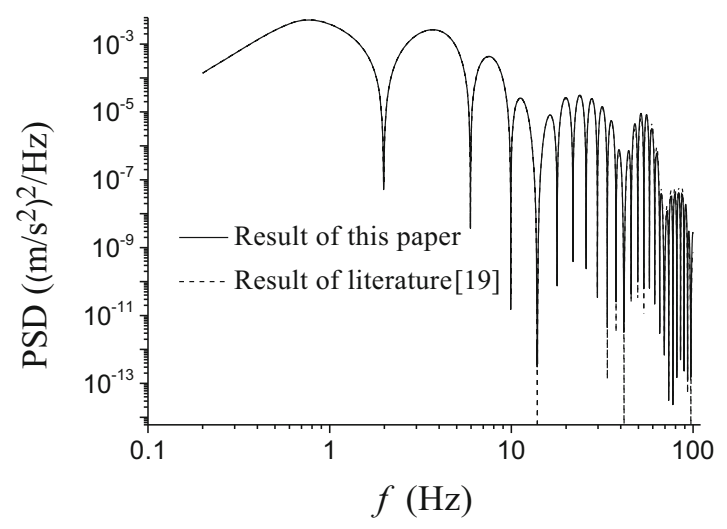

(b) Vertical acceleration of the car body

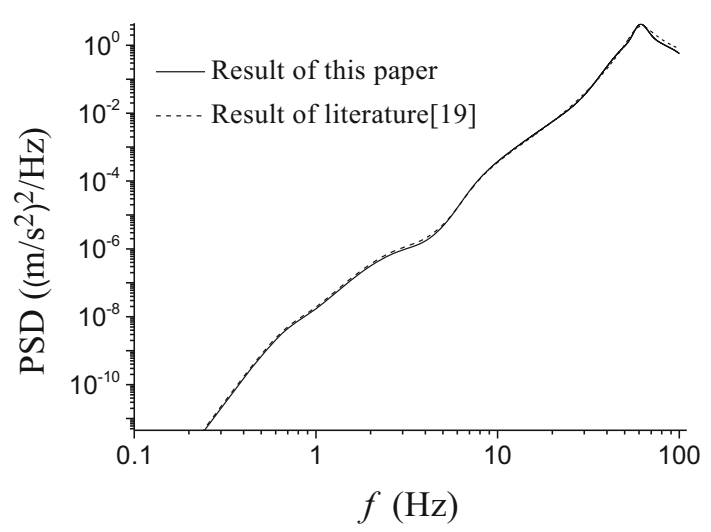

(d) Vertical acceleration of the rail

Fig. 3 Comparison of calculation results 


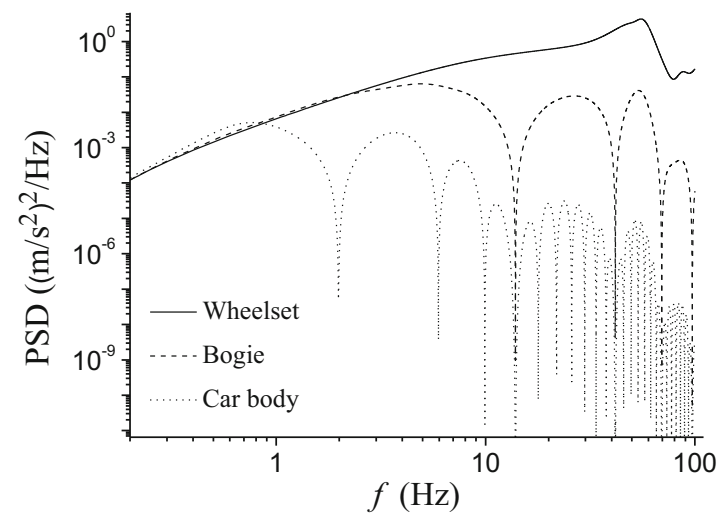

(a) Vertical acceleration of the vehicle

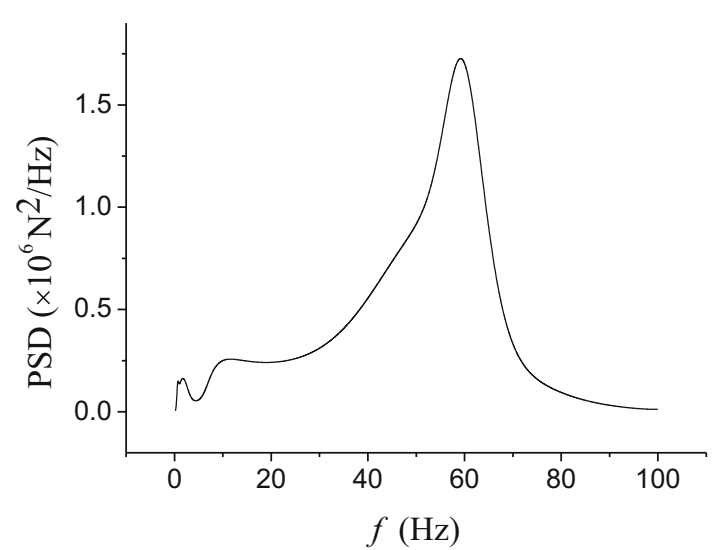

(c) Wheel-rail vertical force

Fig. 4 Frequency response of the vehicle-track coupled system

$\boldsymbol{S}_{y}=\boldsymbol{H}^{*} \boldsymbol{S}_{\mathrm{ir}} \boldsymbol{H}^{\mathrm{T}}$,

where $S_{y}$ is the response spectrum of the system; $S_{\text {ir }}$ is the track irregularity spectrum; $\boldsymbol{H}^{*}$ is the conjugate matrix of $\boldsymbol{H}$; and $\boldsymbol{H}^{\mathrm{T}}$ is the transposed matrix of $\boldsymbol{H}$.

\section{Distribution characteristics of the frequency response of the vehicle-track vertical coupled system}

\subsection{Calculation parameters}

In simulation, the vehicle speed is $250 \mathrm{~km} / \mathrm{h}$. Track irregularity follows the German low-interference spectrum expressed as

$\left.S_{\mathrm{v}}(\Omega)=\frac{A_{\mathrm{v}} \Omega_{\mathrm{c}}^{2}}{\left(\Omega^{2}+\Omega_{\mathrm{r}}^{2}\right)+\left(\Omega^{2}+\Omega_{\mathrm{c}}^{2}\right)}\left(\mathrm{m}^{2} / \mathrm{rad} / \mathrm{m}\right)\right)$,

where parameters are taken as $A_{\mathrm{v}}=4.032 \times 10^{-7}$, $\Omega_{\mathrm{c}}=0.8246$, and $\Omega_{\mathrm{r}}=0.0206$. The frequency range is

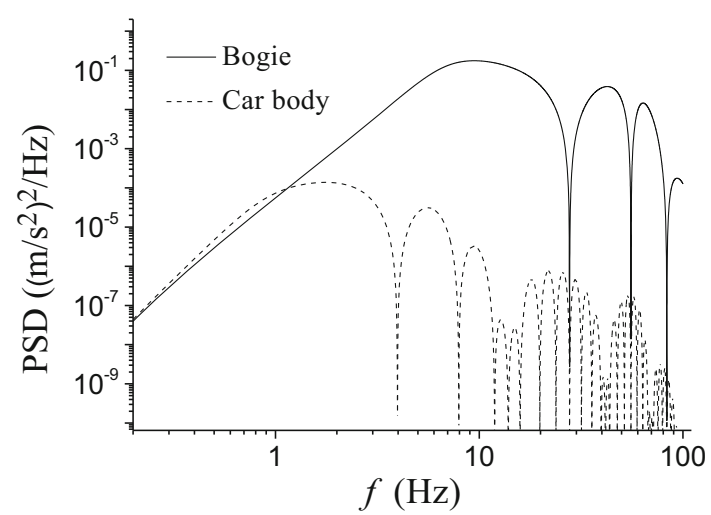

(b) Pitching acceleration of the vehicle

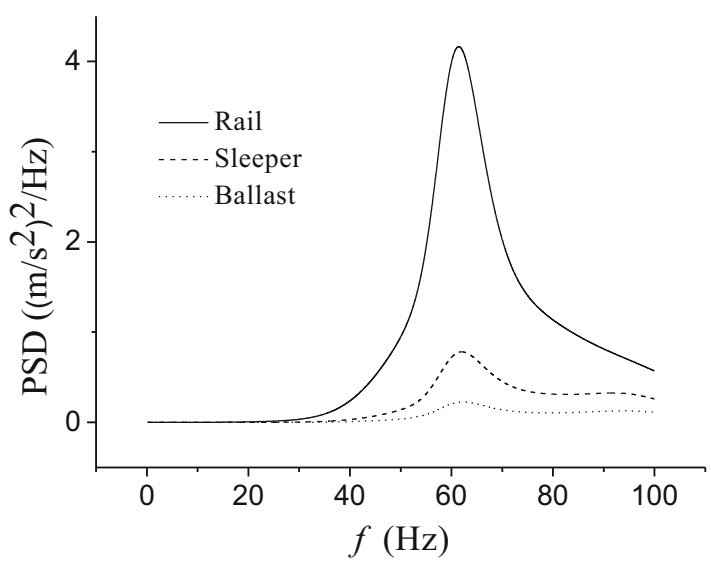

(d) Vertical acceleration of the track

0.2-100 Hz. The vehicle parameters are presented in Table 1. Track parameters are from annexed Table 13 of Ref. [9].

\subsection{Model verification}

To verify the model, we compare the results calculated using the method proposed in this paper with those obtained in Ref. [19], as shown in Fig. 3, where PSD stands for power spectral density. From many dynamic indicators of the vehicle-track coupled system, several typical indicators are selected and presented in the figure. It is evident that the calculation results of the two methods have good consistency, demonstrating the effectiveness of the proposed method.

\subsection{Frequency response of the vehicle-track vertical coupled system}

Figure 4 shows the frequency responses of the vehicletrack vertical coupled system. 


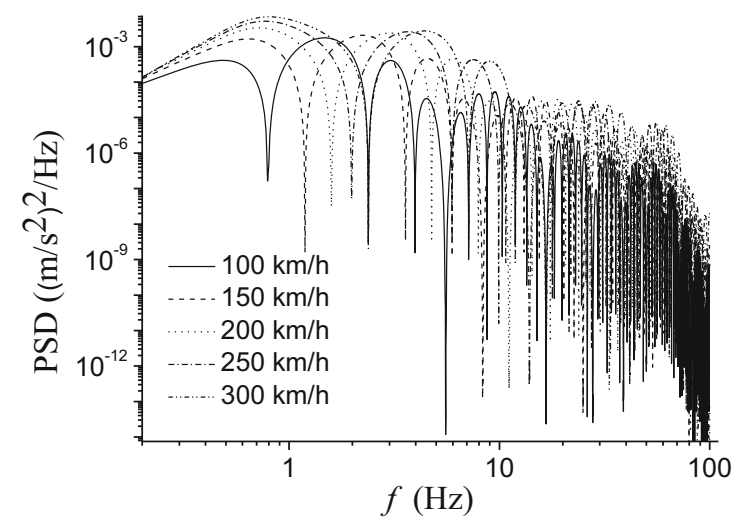

(a) Vertical acceleration of car body

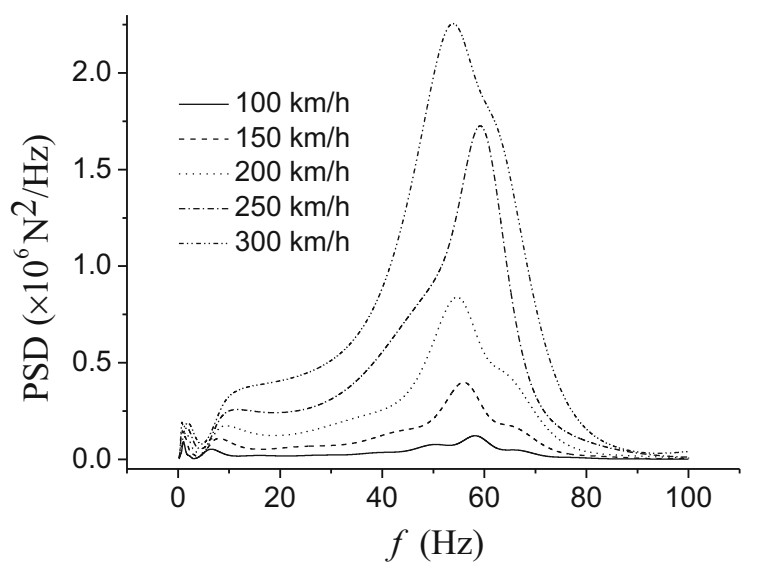

(c) Wheel-rail vertical force

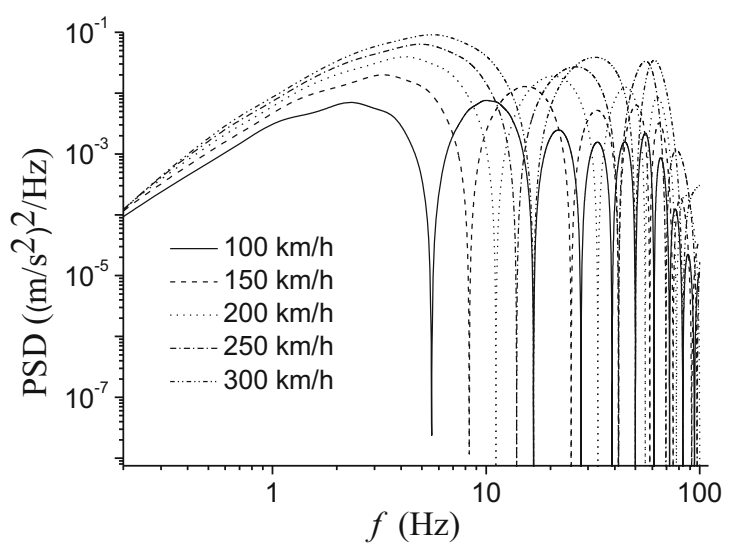

(b) Vertical acceleration of bogie

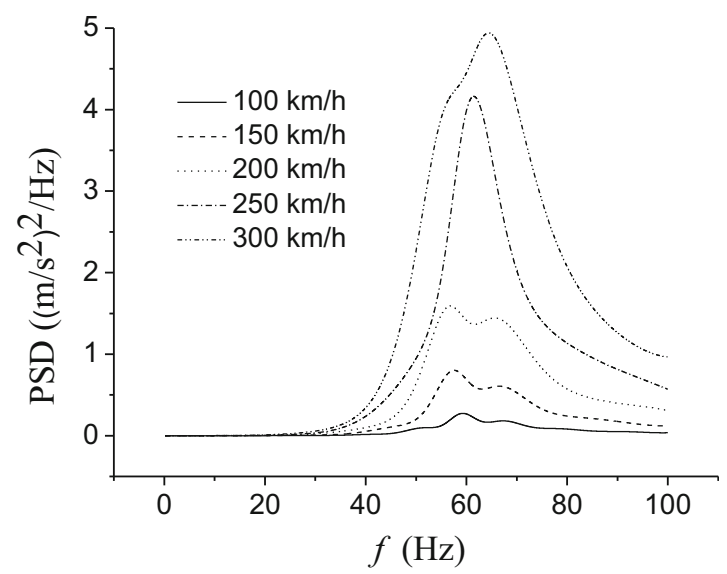

(d) Vertical acceleration of the rail

Fig. 5 Effect of the vehicle speed on the frequency response of the vehicle-track coupled system

Table 2 Vehicle suspension stiffness (MN/m)

\begin{tabular}{llllll}
\hline Case no. & 1 & 2 & 3 & 4 & 5 \\
\hline Primary stiffness & 1.0 & 2.0 & 3.0 & 4.0 & 5.0 \\
Secondary stiffness & 0.1 & 0.4 & 0.7 & 1.0 & 1.2 \\
\hline
\end{tabular}

Figure 4 shows that, within the frequency range analyzed, there are numerous alternating peaks and valleys in the vertical vibration of the car body and bogie. The peaks and valleys are mainly caused by mutual interference of the four wheelsets. If two wheelsets of the bogie move in the same direction, the bogie has vertical motion and no pitching motion; if the wheelsets move in opposite directions, the bogie has pitching motion and no vertical motion. Similarly, if two bogies move in the same direction, the car body has vertical motion and no pitching motion; if two bogies move in opposite directions, the car body has pitching motion and no vertical motion. This phenomenon is known as a geometric filter.

According to the phenomenon of the geometric filter, we know that certain wavelengths of track irregularity do not cause vibrations of the car body and bogie. As a result, vibration valleys form at certain frequencies and peaks form between two neighboring valleys accordingly. The relationships between these wavelengths and the length between bogie pivot centers (i.e., the fixed axle spacing) are

$$
\begin{aligned}
\lambda_{\mathrm{c} i} & =\frac{2 l_{\mathrm{c}}}{2 i-1}(\text { Vertical motion of carbody }), \\
\lambda_{\mathrm{c} j} & =\frac{l_{\mathrm{c}}}{j}(\text { Pitching movement of carbody }), \\
\lambda_{\mathrm{t} i} & =\frac{2 l_{\mathrm{t}}}{2 i-1}(\text { Vertical motion of bogie }), \\
\lambda_{\mathrm{t} j} & =\frac{l_{\mathrm{t}}}{j}(\text { Pitching movement of bogie }),
\end{aligned}
$$




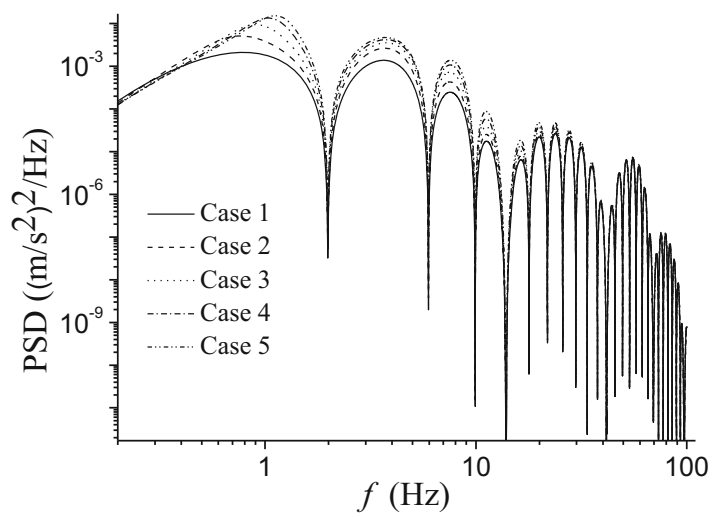

(a) Vertical acceleration of the car body

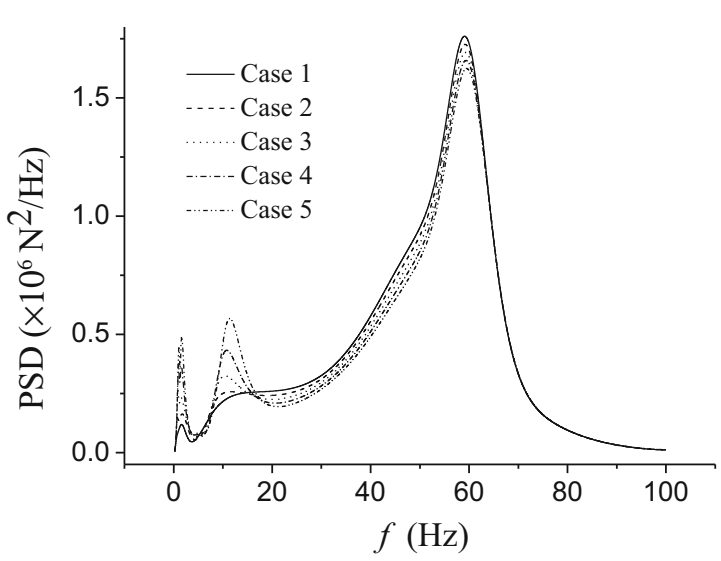

(c) Wheel-rail vertical force

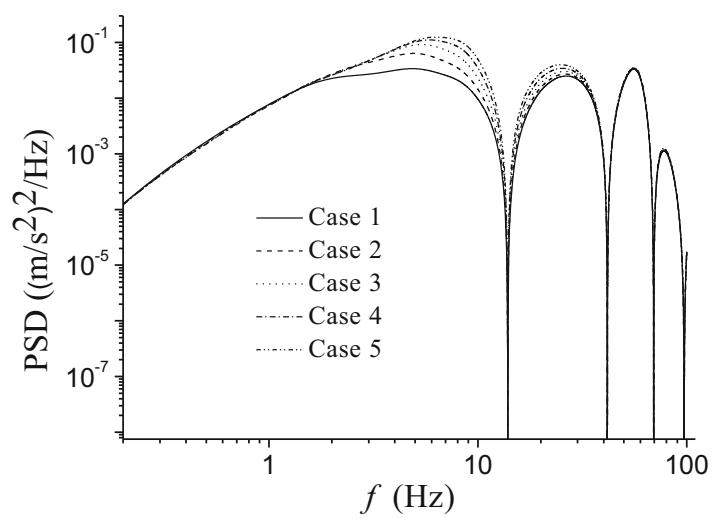

(b) Vertical acceleration of the bogie

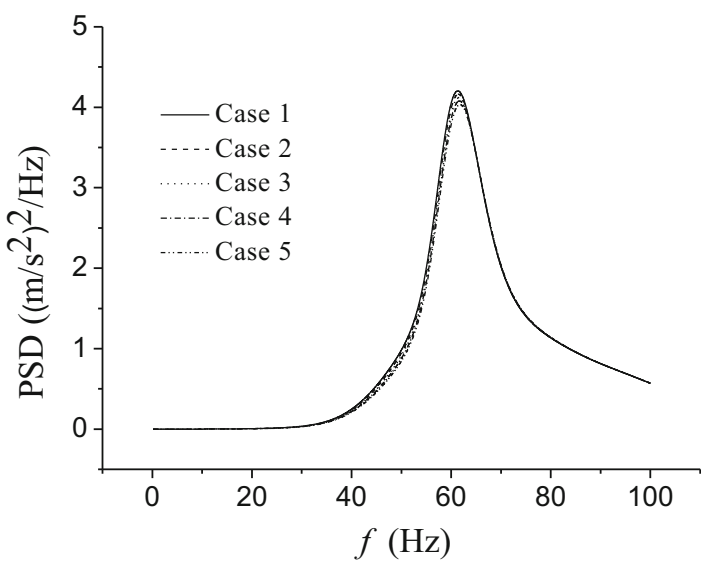

(d) Vertical acceleration of the rail

Fig. 6 Effect of the vehicle suspension stiffness on the frequency response of the vehicle-track vertical coupled system

Table 3 Vehicle suspension damping $(\mathrm{kN} \cdot \mathrm{s} / \mathrm{m})$

\begin{tabular}{llllll}
\hline Case no. & 1 & 2 & 3 & 4 & 5 \\
\hline Primary damping & 20 & 50 & 80 & 100 & 120 \\
Secondary damping & 50 & 100 & 150 & 200 & 250 \\
\hline
\end{tabular}

where $l_{\mathrm{c}}$ is the length between bogie pivot centers; $l_{\mathrm{t}}$ is the fixed axle spacing; $\lambda_{c i}$ is the wavelength corresponding to the $i$ th valley of the vertical acceleration of the car body; $\lambda_{c j}$ is the wavelength corresponding to the $j$ th valley of the pitching acceleration of the car body; $\lambda_{t i}$ is the wavelength corresponding to the $i$ th valley of the vertical acceleration of the bogie; and $\lambda_{\mathrm{t} j}$ is the wavelength corresponding to the $j$ th valley of the pitching acceleration of the bogie.

Under the excitation of the German low-interference spectrum, the vertical vibration of the car body is mainly in the low-frequency band, and that of the bogie has a wide frequency distribution, being strong from several Hertz to dozens of Hertz. Meanwhile, the vertical vibration of the wheelset, wheel-rail force, and track structure are weak in the low-frequency range and strong at a frequency of dozens of Hertz.

\section{Factors affecting the frequency response of the vehicle-track coupled system}

The vehicle speed, vehicle suspension parameters, and track support parameters strongly affect the vibration of the vehicle-track coupled system. This section analyzes the effects of the vehicle speed, vehicle suspension parameters, and track support parameters on the vehicle-track coupled system.

\subsection{Effect of the vehicle speed}

The effect of the vehicle speed on the frequency response of the vehicle-track coupled system is shown in Fig. 5. Given that there are too many dynamic indicators of the 


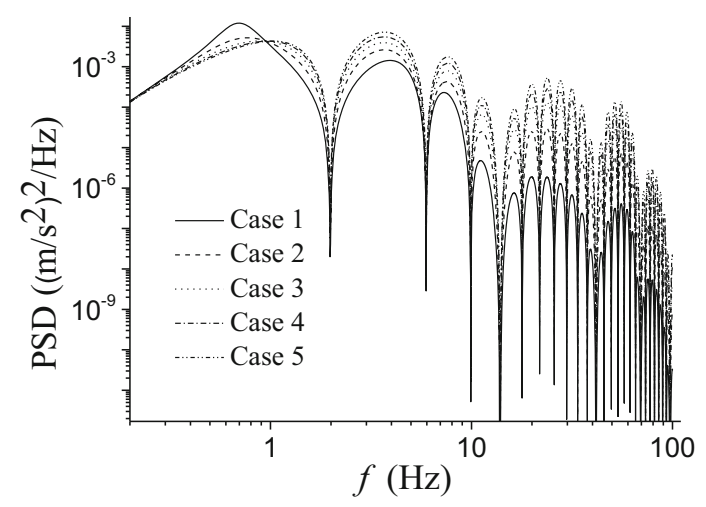

(a) Vertical acceleration of the car body

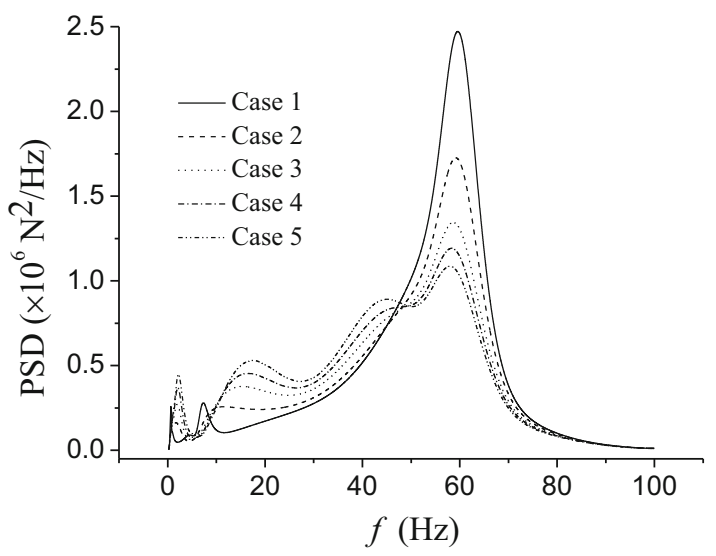

(c) Wheel-rail vertical force

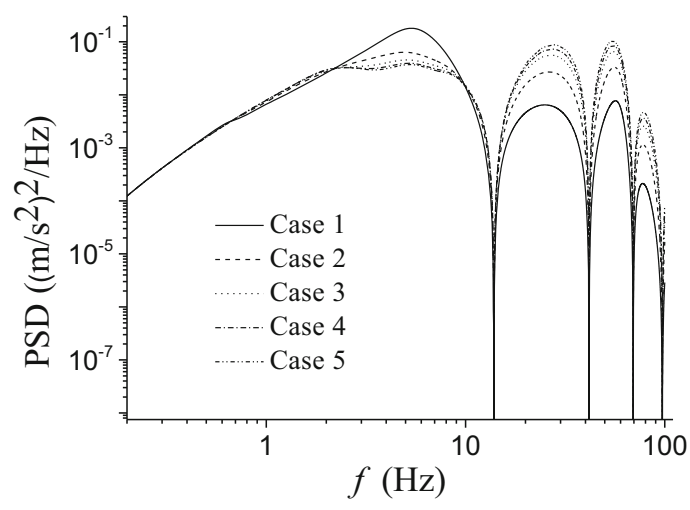

(b) Vertical acceleration of the bogie

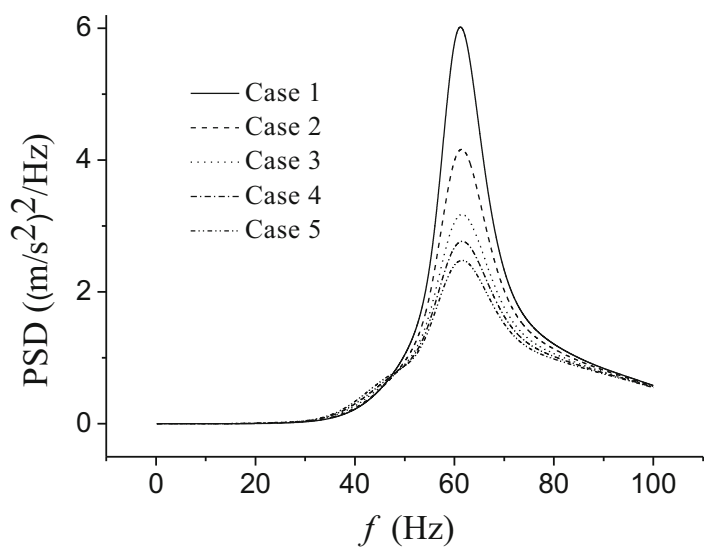

(d) Vertical acceleration of the rail

Fig. 7 Effect of vehicle suspension damping on the frequency response of the vehicle-track coupled system

Table 4 Cases of track stiffness $(\mathrm{MN} / \mathrm{m})$

\begin{tabular}{lllll}
\hline Case no. & 1 & 2 & 3 & 4 \\
\hline Vertical stiffness of fasteners & 40 & 80 & 120 & 160 \\
Vertical stiffness of ballast & 150 & 200 & 250 & 300 \\
Vertical stiffness of subgrade & 150 & 200 & 250 & 300 \\
\hline
\end{tabular}

vehicle-track coupled system, only four are presented: vertical accelerations of the car body, bogie, rail, and the wheel-rail vertical force.

Figure 5 shows that, with an increase in vehicle speed, the vertical vibration of the vehicle-track coupled system increases, and the vertical vibration of the car body and bogie obviously shifts to higher frequency.

\subsection{Effects of vehicle suspension parameters}

The car body, bogie, and wheelset are linked by suspension systems, and suspension parameters also strongly affect the vibration of the coupled system. This section analyzes the effects of primary and secondary suspension stiffness and damping on the frequency response of the vehicle-track vertical coupled system.

\subsubsection{Effect of the vehicle suspension stiffness}

The calculation cases of the vehicle system suspension stiffness are presented in Table 2. The effect of the vehicle suspension stiffness on the frequency response of the vehicle-track vertical coupled system is shown in Fig. 6.

Figure 6 shows that, with an increase in vehicle suspension stiffness, the vertical vibrations of the car body and bogie at a frequency lower than $40 \mathrm{~Hz}$ decrease and those higher than $40 \mathrm{~Hz}$ are basically unchanged. The vertical vibration of the wheel-rail force increases at a frequency lower than $15 \mathrm{~Hz}$, slightly decreases within the range of $15-65 \mathrm{~Hz}$, and is basically remained unchanged above $65 \mathrm{~Hz}$. Increasing the vehicle suspension stiffness thus increases the low-frequency vertical vibration of the vehicle system and wheel-rail vertical force. 


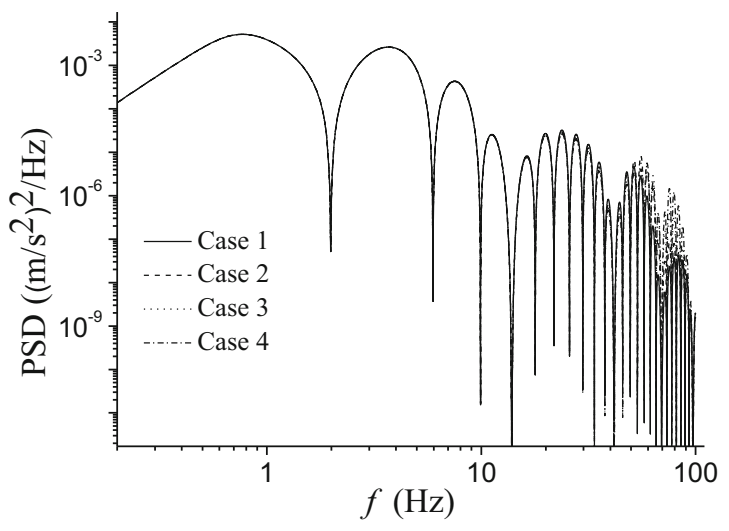

(a) Vertical acceleration of the car body

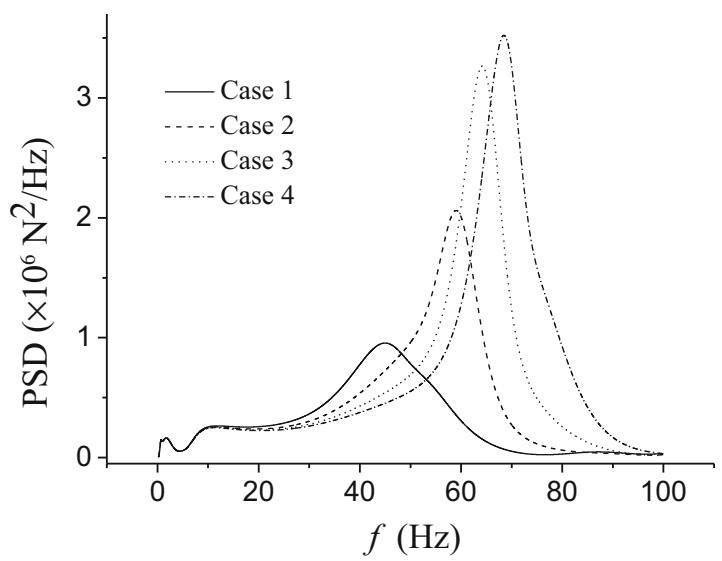

(c) Wheel-rail vertical force

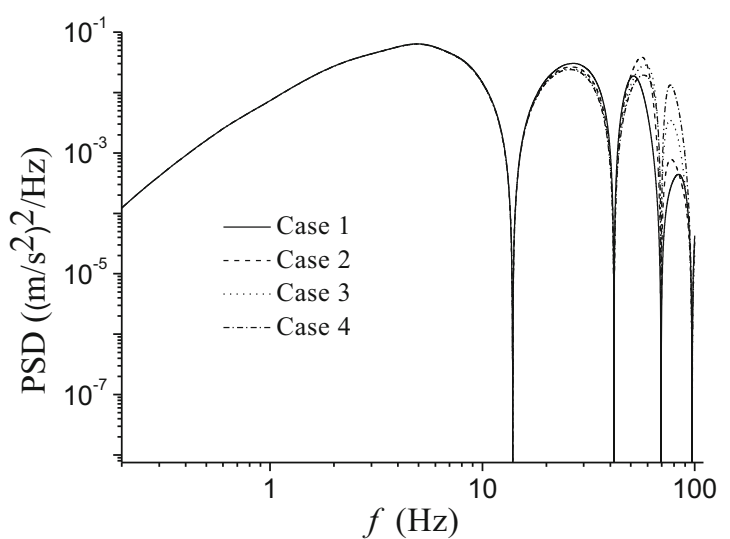

(b) Vertical acceleration of the bogie

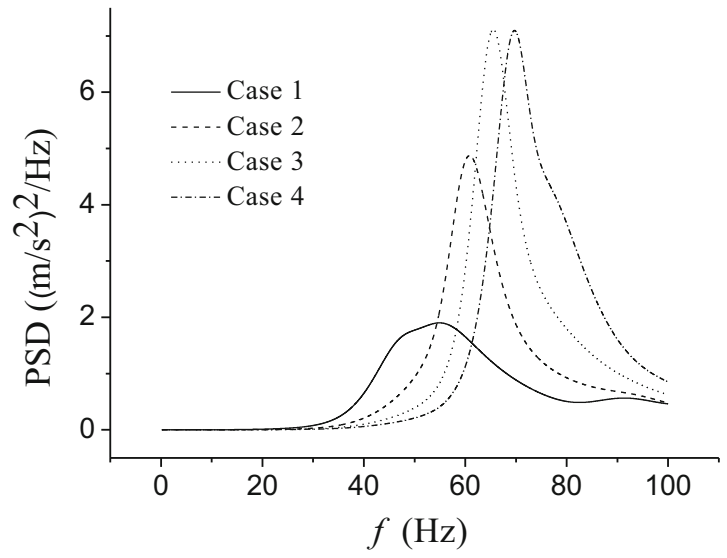

(d) Vertical acceleration of the rail

Fig. 8 Effect of track stiffness on the frequency response of the vehicle-track vertical coupled system

Table 5 Cases of track damping $(\mathrm{kN} \cdot \mathrm{s} / \mathrm{m})$

\begin{tabular}{lllll}
\hline Case no. & 1 & 2 & 3 & 4 \\
\hline Fastener damping & 60 & 80 & 100 & 120 \\
Ballast damping & 30 & 60 & 90 & 120 \\
Subgrade damping & 30 & 60 & 90 & 120 \\
\hline
\end{tabular}

\subsubsection{Effect of vehicle suspension damping}

The calculation cases of vehicle suspension damping are presented in Table 3. The effect of vehicle suspension damping on the frequency response of the vehicle-track coupled system is shown in Fig. 7.

Figure 7 shows that, with an increase in vehicle suspension damping, the vertical vibration of the car body decreases at a frequency lower than $1 \mathrm{~Hz}$ and increases above $1 \mathrm{~Hz}$. The vertical vibration of the bogie decreases at a frequency lower than $10 \mathrm{~Hz}$ and increases above $10 \mathrm{~Hz}$. The vertical vibration of the wheel-rail force increases at a frequency lower than $50 \mathrm{~Hz}$, appreciably decreases within the range of 50-80 Hz, and slightly decreases above $80 \mathrm{~Hz}$. The vertical vibration of the rail decreases within the range of $50-80 \mathrm{~Hz}$. An increase in vehicle suspension damping thus effectively reduces the low-frequency vertical vibration of the car body and bogie as well as the vertical vibration of the wheel-rail force and track structure at $50-80 \mathrm{~Hz}$, but is unfavorable in terms of mid-frequency and high-frequency vertical vibrations of the vehicle system.

\subsection{Effects of track support parameters}

Track stiffness is an important parameter for the design of a railway track structure. With the operation of a railway, a rail pad will age and harden and ballast will be gradually affected. These factors will lead to changes in the stiffness and damping of the track support. This section analyzes the effects of track stiffness and damping on the frequency response of the vehicle-track coupled system. 


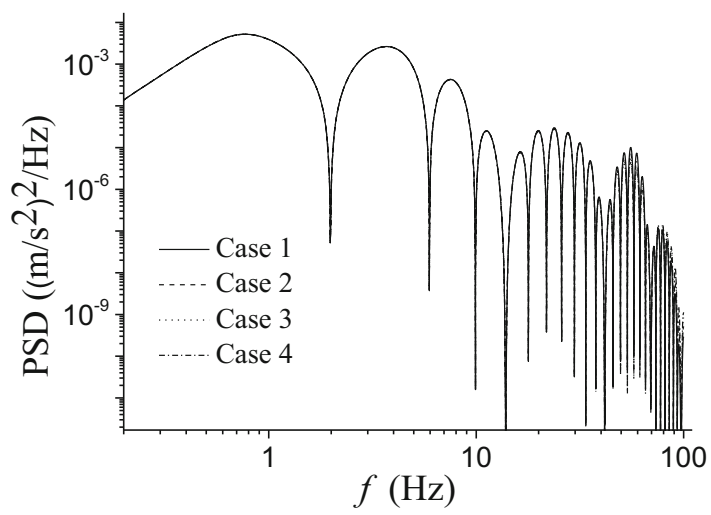

(a) Vertical acceleration of the car body

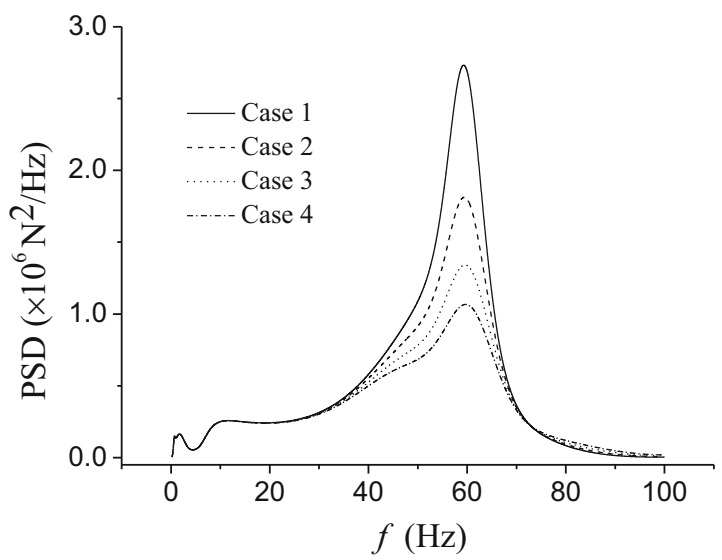

(c) Wheel-rail vertical force

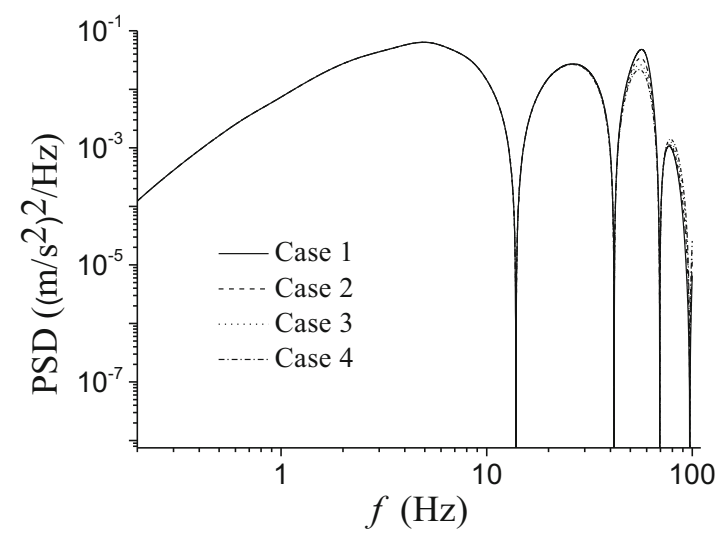

(b) Vertical acceleration of the bogie

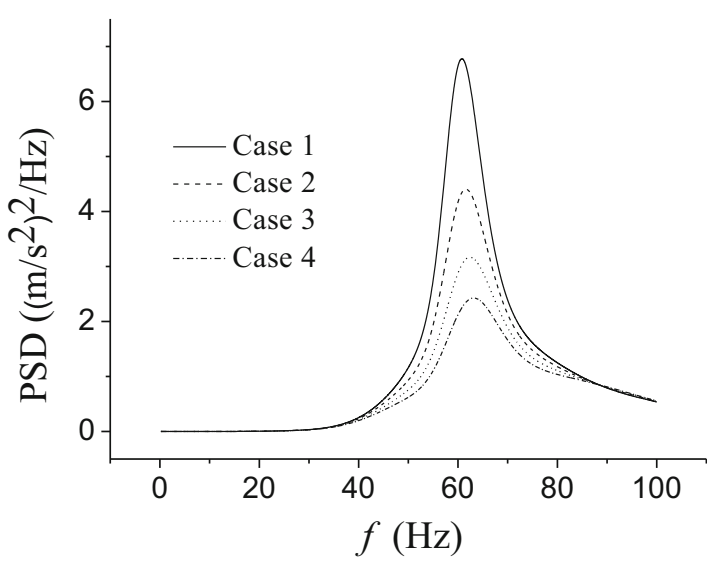

(d) Vertical acceleration of the rail

Fig. 9 Effect of track damping on the frequency response of the vehicle-track vertical coupled system

\subsubsection{Effect of track stiffness}

The calculation cases of track stiffness are presented in Table 4. The effect of track stiffness on the frequency response of the vehicle-track coupled system is shown in Fig. 8.

Figure 8 shows that, with an increase in track stiffness, the vertical vibrations of the car body and bogie are basically unchanged at a frequency lower than $15 \mathrm{~Hz}$, slightly decrease within the range of $15-50 \mathrm{~Hz}$, and increase above $50 \mathrm{~Hz}$. However, the vertical vibration of the car body is mainly in the low-frequency range, and it can thus be considered that track stiffness has little effect on the vertical vibration of the car body. The vertical vibrations of the rail and wheel-rail force increase with an increase in track stiffness. It is thus evident that track stiffness has little effect on the vertical vibration of the car body, but the vertical vibrations of the bogie, wheel-rail force, and track structure increase with an increase in track stiffness.

\subsubsection{Effect of track damping}

The calculation cases of track damping are presented in Table 5. The effect of track damping on the frequency response of the vehicle-track coupled system is shown in Fig. 9.

Figure 9 shows that, with an increase in track damping, the vertical vibrations of the car body and bogie are basically unchanged at a frequency lower than $35 \mathrm{~Hz}$, slightly decrease within the range of $35-70 \mathrm{~Hz}$, and slightly increase above $70 \mathrm{~Hz}$. It is thus considered that track damping has little effect on the vertical vibrations of the car body and bogie. The vertical vibrations of the wheelrail force and rail decrease within the range of $40-70 \mathrm{~Hz}$ and change little at other frequencies. It is thus evident that an increase in track damping effectively reduces the vertical vibration of the wheel-rail force and track structure but has little effect on the vertical vibrations of the car body and bogie. 


\section{Conclusions}

On the basis of the theory of vehicle-track coupled dynamics, the frequency response of the vehicle-track vertical coupled system was calculated by combining frequency analysis and symplectic mathematics methods, and the effects of vehicle speed, vehicle suspension parameters, and track support parameters on the frequency response of the coupled system were studied.

(1) Mutual interference between four wheelsets causes the phenomenon of the geometrical filter, resulting in numerous alternating peaks and valleys in the vibrations of the car body and bogie. The vertical vibration of the car body is mainly in the low-frequency band while that of the bogie has a wide frequency distribution, being strong from several Hertz to dozens of Hertz. The vertical vibrations of the wheel-rail force, wheelset, and track structure mainly occur at a frequency of dozens of Hertz.

(2) With an increase in vehicle speed, the vertical vibration of the vehicle-track coupled system increases while the vertical vibrations of the car body and bogie shift to higher frequency.

(3) With an increase in vehicle suspension stiffness, the low-frequency vibrations of the vehicle system and wheel-rail vertical force increase while the vertical vibration of the track structure has little change. An increase in vehicle suspension damping can effectively reduce the low-frequency vibrations of the car body and bogie as well as the vibrations of the wheelrail vertical force and track structure within the frequency range of 50-80 Hz, but mid-frequency and high-frequency vibrations of the vehicle system increase.

(4) Track stiffness has little effect on the vibration of the car body, but the vibrations of the bogie, wheel-rail force, and track structure increase with an increase in track stiffness. An increase in track damping can effectively reduce the vertical vibrations of the wheel-rail vertical force and track structure, but has little effect on the vibrations of the car body and bogie.

Open Access This article is distributed under the terms of the Creative Commons Attribution 4.0 International License (http:// creativecommons.org/licenses/by/4.0/), which permits unrestricted use, distribution, and reproduction in any medium, provided you give appropriate credit to the original author(s) and the source, provide a link to the Creative Commons license, and indicate if changes were made.

\section{References}

1. Luo L, Zhang GM, Wu WQ (2000) Control of track irregularity of wheel-rail system [M]. China Railway Publishing House, Beijing (in Chinese)

2. Luo L (1982) Track random excitation functions[J]. China Railway Sci 3:74-112 (in Chinese)

3. Chen G (2000) The analysis on random vibration of vehicle/track coupling system[D]. Southwest Jiaotong University, Chengdu (in Chinese)

4. Zhai WM (2007) Vehicle-Track Coupling Dynamics[M]. Science Press, Beijing (in Chinese)

5. Lei X, Noda NA (2002) Analyses of dynamic response of vehicle and track coupling system with random irregularity of track vertical profile[J]. J Sound Vib 258:147-165

6. Fang F (1995) Engineering random vibration [M]. National Defense Industry Press, Beijing (in Chinese)

7. Jin XU, Cang-ru JIANG, Wei-ping XIE (2007) Research on track structure of wuhan light rail based on the random vibration theory [J]. J Wuhan Univ Technol 29(6):100-103 (in Chinese)

8. Lei XY (2007) Dynamic analyses of track structure with Fourier transform technique [J]. J China Railway Soc 29(3):67-71 (in Chinese)

9. Wang KY, Cai CB, Xu ZS (2005) Analysis of random vibration characteristics and verification of track structure on frequency domain method [J]. Chin J Mech Eng 41(11):149-157 (in Chinese)

10. Lin JH, Zhang YH (2004) Pseudo excitation method in random vibration[M]. Science Press, Beijing (in Chinese)

11. Lu F, Kennedy D, Williams FW, Lin JH (2008) Symplectic analysis of vertical random vibration for coupled vehicle-track systems[J]. J Sound Vib 317:236-249

12. Zhang YW, Lin JH, Zhao Y, Howson WP, Williams FW (2010) Symplectic random vibration analysis of a vehicle moving on an infinitely long periodic track [J]. J Sound Vib 329:4440-4454

13. Zhang YW, Zhao Y, Zhang YH et al (2013) Riding comfort optimization of railway trains based on pseudo-excitation method and symplectic method [J]. J Sound Vib 332(21):5255-5270

14. Zhang J, Zhao Y, Zhang Y et al (2013) Non-stationary random vibration of a coupled vehicle-slab track system using a parallel algorithm based on the pseudo excitation method [J]. Proc Inst Mech Eng F 227(3):203-216

15. Zeng J, Luo R (2007) Vibration analysis of railway passenger car systems by considering flexible carbody effect[J]. J China Railway Soc 29:19-25 (in Chinese)

16. Wu PB, Zeng J (2004) Dynamic response analysis of railway passenger car with flexible carbody model based on the semiactive suspensions[J]. Veh Syst Dyn 41:774-783

17. Zhou JS (2012) Vibration and control of railway vehicles[M]. China Railway Publishing House, Beijing (in Chinese)

18. Zhou JS, Gong D, Sun WJ et al (2009) Influence of vertical elasticity of carbody of railway passenger vehicles on ride quality[J]. J China Railway Soc 31(2):32-37 (in Chinese)

19. Coenraad C (2001) Modern railway track[M]. MRT-Productions, Lithonia 